\title{
American Society of Mechanical Engineers
}

ASME Accepted Manuscript Repository

Institutional Repository Cover Sheet

Cranfield Collection of E-Research - CERES

ASME Paper

Title: $\quad$ Comparison of hydrogen micromix flame transfer functions determined using RANS and LES

Jonathan McClure, David Abbott, Parash Agarwal, Xiaoxiao Sun, Giulia Babazzi, Vishal Sethi,

Authors:

Pierre Gauthier

ASME Conf Title: ASME Turbo Expo 2019

Volume/Issue: Volume 3, Paper GT2019-90538

Date of Publication (VOR* Online) 5 November 2019

ASME Digital Collection https://asmedigitalcollection.asme.org/GT/proceedings/GT2019/58608/Phoenix,\%20Arizona, URL:

\%20USA/1066620

DOI: $\quad$ https://doi.org/10.1115/GT2019-90538

*VOR (version of record) 
June 17-21, 2019, Phoenix, Arizona, USA

GT2019-90538

\section{COMPARISON OF HYDROGEN MICROMIX FLAME TRANSFER FUNCTIONS DETERMINED USING RANS AND LES}

\author{
Jonathan McClure, David Abbott, Parash Agarwal, \\ Xiaoxiao Sun, Giulia Babazzi, Vishal Sethi \\ Centre for Propulsion Engineering \\ Cranfield University \\ Cranfield, Bedfordshire, MK43 OAL, UK
}

Pierre Q. Gauthier ${ }^{\dagger}$

Royal Academy of Engineering

Visiting Professor in Low Emissions Combustion System Modelling

Cranfield University

Cranfield, Bedfordshire, MK43 OAL, UK

\begin{abstract}
Hydrogen has been proposed as an alternative fuel to meet long term emissions and sustainability targets, however due to the characteristics of hydrogen significant modifications to the combustion system are required. The micromix concept utilises a large number of miniaturised diffusion flames to improve mixing, removing the potential for local stoichiometric pockets, flashback and autoignition. No publicly available studies have yet investigated the thermoacoustic stability of these combustion systems, however due to similarities with lean-premixed combustors which have suffered significant thermoacoustic issues, this risk should not be neglected.

Two approaches have been investigated for estimating flame response to acoustic excitations of a single hydrogen micromix injector element. The first uses analytical expressions for the flame transfer function with constants obtained from RANS CFD while the second determines the flame transfer function directly using unsteady LES CFD. Results show the typical form of the flame transfer function but suggest micromix combustors may be more susceptible to higher frequency instabilities than conventional combustion systems. Additionally, the flame transfer function estimated using RANS CFD is broadly similar to that of the LES approach, therefore this may be suitable for use as a preliminary design tool due to its relatively low computational expense.
\end{abstract}

\footnotetext{
*Address all correspondence to this author (email: mcclure@td.mw.tum.de).

${ }^{\dagger}$ Also a Senior Key Combustion Expert at Siemens Energy, Dorval, QC, H9P 1A5, Canada
}

\author{
NOMENCLATURE \\ Acronyms \\ CFD Computational Fluid Dynamics \\ FDF Flame Describing Function \\ FFT Fast Fourier Transform \\ FGM Flamelet Generated Manifold \\ FTF Flame Transfer Function \\ LES Large Eddy Simulation \\ $N O_{x} \quad$ Nitrogen Oxides \\ $\mathrm{OH}$ Hydroxyl Radical \\ PDF Probability Density Function \\ RANS Reynolds Averaged Navier Stokes \\ Latin Symbols \\ A Perturbation Amplitude [-] \\ c Progress Variable \\ f Frequency [Hz] \\ n Proportionality Constant [-] \\ Q Heat Release Rate [W] \\ u Velocity $[\mathrm{m} / \mathrm{s}]$

\section{Greek Symbols} \\ $\Delta \tau \quad$ Spread of Time Delays [s] \\ $\theta \quad$ Time Delay Variation Parameter [-] \\ $\tau \quad$ Time Delay [s] \\ $\varphi$ Equivalence Ratio [-] \\ $\phi \quad$ Phase Difference [rad] \\ $\omega$ Angular frequency $[\mathrm{rad} / \mathrm{s}]$
}




\section{INTRODUCTION}

The need for new aero-engine technologies is primarily driven by the requirement to meet progressively restrictive emissions regulations while remaining cost effective for engine operators [1]. In order to remain compliant with these regulations aircraft gas turbine engines have continuously evolved since their inception to improve efficiency and reduce fuel burn [2]. The combustor is the most critical component in determining the environmental impact of an engine and has undergone significant changes to reduce aviation emissions generation [3], however meeting long term emissions targets will require a move to alternative fuels [4]. Changing from the use of kerosene is also necessary from a sustainability perspective due to diminishing fossil fuel reserves [5].

One of the most promising alternative fuels for future aircraft to meet long term emissions targets is hydrogen. This will require a significant redesign of the engine, as well as the overall aircraft [6], due to the considerably different characteristics of hydrogen compared to kerosene [7]. This is also an area of interest for land-based applications as Mitsubishi Hitachi Power Systems (MHPS) recently developed a large scale gas turbine with co-firing capabilities for natural gas and hydrogen content up to $30 \%$, with plans to develop a fully hydrogen powered engine utilising a cluster combustor with many small premixed flames [8].

Hydrogen combustion is characterised by high flame speeds and high stoichiometric flame temperatures, therefore conventional fuel injectors are not suitable for use with hydrogenfuelled gas turbines due to the risk of flashback and high temperature hotspots resulting from imperfect mixing [9]. The micromix concept has been developed to address these issues and exploit the physical properties of hydrogen, allowing smaller, lower emissions combustors [10]. Micromix injectors consist of a large number of individual injection elements which results in a series of small micro-diffusion flames. Each injector element includes an air guiding panel (AGP), shown in Fig. 1, which accelerates the airflow and creates large recirculation regions downstream of the injector. These recirculation zones anchor the flames and are the main source of flame stability and separation from the surrounding flames. Fuel is injected in a cross-flow jet perpendicular to the air stream which greatly improves the mixing characteristics [11]. The discrete injection of fuel and air provides inherent safety against flashback and autoignition while the improved mixing from the jet in cross-flow reduces the potential for stoichiometric regions due to poor fuel/air mixing.

When making substantial changes to combustor designs, the potential to alter the thermoacoustic properties must be considered to avoid potentially destructive combustion oscillations. Historically, gas turbine engines with conventional diffusion combustors have not suffered major issues with thermoacoustic instabilities [12]. With the introduction of lean-premixed (LP) combustors there have been significant and expensive incidents caused by combustion-acoustic coupled pressure oscilla- tions [13]. There are also examples of instabilities arising from changes in fuel source, such as from typical natural gas to imported liquefied natural gas (LNG) [14]. It is therefore essential to consider thermoacoustics from early in the development process as if left unaddressed this could result in expensive redesigns in later development stages if significant stability issues arise.

At present, no publicly available studies have been conducted investigating the thermocoustic characteristics of the hydrogen micromix concept. This may be due in part to the assumption that because these systems have diffusion flames, which have historically been more stable than premixed flames, this should prove beneficial for thermoacoustic stability and result in a low risk of serious thermoacoustic instabilities occurring. Despite this, the potential for combustion oscillations cannot be neglected, particularly due to the lean combustion nature of the concept, resulting in less acoustic damping due to the reduced amount of cooling air. Additional factors such as the influence of injector-injector interaction resulting from the large number of injection elements and the impact of the large number of holes on combustor acoustics, which could potentially lead to amplification of oscillations [15], will also produce significantly different behaviour from conventional diffusion based systems.

The sensitivity of thermoacoustic instabilities to changes in operating conditions, combustor geometry and fuel type requires in-depth investigation which is a costly and time consuming process and was generally neglected for early combustor design. To minimise experimental tests there has been growing interest in predicting the stability of combustors using CFD combined with low-order models [16]. These methods rely on a computationally or experimentally obtained flame model in the form of a transfer function describing the response of the flame to upstream perturbations in the air or fuel flows. There are two main forms of this transfer function; the flame transfer function (FTF) which describes the flame response to small inlet flow perturbations, and the flame describing function (FDF) which can capture the decrease in flame amplification factor with increasingly large amplitude inlet flow perturbations. By combining these flame models with low-order acoustic models of the combustor, thermoacoustic properties can be analysed without requiring a series of expensive and time intensive experimental tests.

The aim of this work is to investigate methods for determining thermoacoustic stability characteristics from a computational approach to augment the development process of hydrogen micromix combustions systems. This is a continuation of the considerable ongoing research efforts at Cranfield University to develop the hydrogen micromix concept [17-26]. FTFs estimated from RANS simulations combined with analytical formulations and FTFs estimated directly from LES simulations are compared to determine the suitability of the low fidelity RANS methods for use as a design tool. Additionally, due to the absence of studies investigating the thermoacoustic characteristics of these combustion systems, this also provides an initial insight 


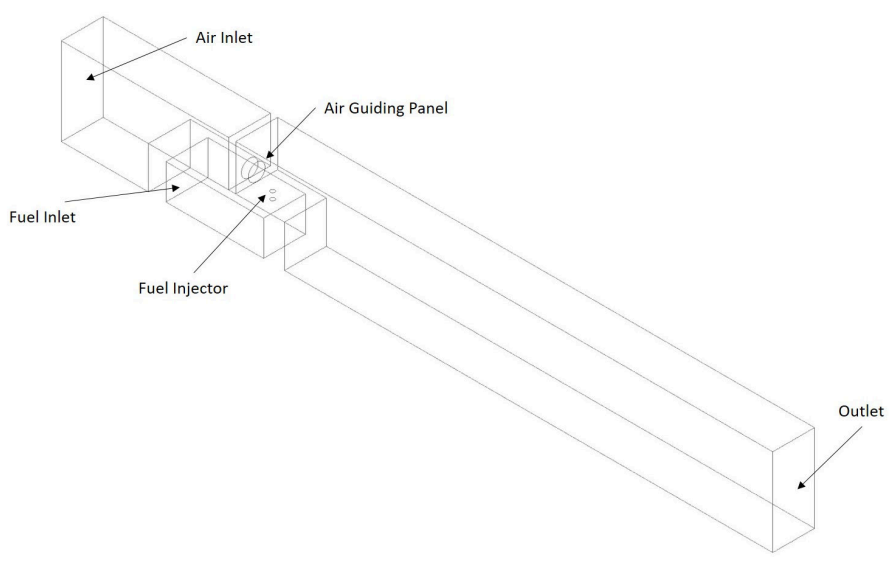

FIGURE 1: THREE DIMENSIONAL GEOMETRIC MODEL SHOWING A SINGLE HYDROGEN MICROMIX INJECTOR ELEMENT

into the likely thermoacoustic behaviour and can assist with the design of experimental test rigs and the continuing development of the hydrogen micromix concept for both aerospace and power generation applications.

\section{NUMERICAL SIMULATUONS}

ANSYS Fluent 18.2 is used for all CFD simulations within this project due to its broad range of well validated physical modelling capabilities. The computational model used is based on previous research at Cranfield University on the design of hydrogen micromix combustion systems using CFD [27]. As experiments on these combustion systems have not yet been conducted at Cranfield University to validate the simulations, the computational model is derived from the configuration investigated by Aachen University which has been validated experimentally [28]. This will allow the preliminary CFD work to inform the initial test rig configuration design and undergo a validation process once experiments have commenced.

For this preliminary investigation into the thermoacoustic characteristics of hydrogen micro-diffusion flames a single injector element is investigated. Fig. 1 shows the geometric model for this injector element. The model takes advantage of the radial symmetry of the injector to the reduce computational expense by simulating half of the real injector geometry using symmetry boundary conditions. In order to maintain the non-premixed characteristics of hydrogen micromix injectors, the air and fuel are introduced through discrete inlets. These air and fuel inlets are positioned sufficiently far upstream of the air guiding panel (AGP) and the fuel injection hole respectively to avoid any influence of the boundaries on the mixing and reaction zones. The orientation of the hydrogen jet from the fuel injection hole per-
TABLE 1: BOUNDARY CONDITION SETTINGS

\begin{tabular}{lll}
\hline Boundary & Variable & Value \\
\hline \multirow{4}{*}{ Air Inlet } & Type & Mass Flow Inlet \\
& Fluid & Air $\left(21 \% \mathrm{O}_{2}+79 \% \mathrm{~N}_{2}\right)$ \\
& Mass Flow Rate $(\mathrm{kg} / \mathrm{s})$ & $1.64 \times 10^{-5}$ \\
& Temperature $(\mathrm{K})$ & 560 \\
& Turbulence Intensity $(\%)$ & 5 \\
\hline \multirow{3}{*}{ Fuel Inlet } & Type & Mass Flow Inlet \\
& Fluid & Hydrogen $\left(\mathrm{H}_{2}\right)$ \\
& Temperature $(\mathrm{K})$ & 300 \\
& Turbulence Intensity $(\%)$ & 5 \\
\hline \multirow{2}{*}{ Domain } & Operating Pressure $(\mathrm{Pa})$ & 101325 \\
\hline
\end{tabular}

pendicular to the air flow through the AGP captures the crossflow properties of the micromix concept.

The fuel and air are injected through discrete mass flow inlets. The mass flow rates of these inlets were calculated to produce an equivalence ratio of $\varphi=0.43$. This equivalence ratio was selected to match the conditions used for a $N O_{x}$ model validation test carried out by Aachen University [29]. The air is preheated to a temperature of $560 \mathrm{~K}$ to reflect thermal conditions downstream of a compressor, however as the experiments were carried out at ambient pressure conditions the operating pressure is set as $101325 \mathrm{~Pa}$. The fuel (pure gaseous hydrogen) enters the combustion chamber at a temperature of $300 \mathrm{~K}$. Turbulence intensity is set at an initial value of $5 \%$ as the upstream configuration is not known at this development stage.

Boundaries which a fluid crosses are defined as interfaces, these are defined at the entry and exit of the AGP and the hydrogen injection hole. The lower and side boundaries are set as walls while the upper surface is a symmetry boundary. This limits this study to a single injector element and will not account for injector-injector interactions, which is an important aspect of micromix combustion systems. A summary of the boundary condition settings is provided in Table 1 .

The combustion process is modelled using the Flamelet Generated Manifold (FGM) approach with diffusion flamelets and the partially premixed model which captures the nonequilibrium effects present in hydrogen micromix flames [24]. A 12 species combustion mechanism with 105 reactions is used to capture the hydrogen/air kinetics, further details of this mechanism and the selection process are provided in [30]. For the 
RANS approach, the $k-\omega$ SST model is used to capture the turbulence effects due to its enhanced treatment of near wall flows and jets and its improved capability of capturing the variability in turbulence scales present due to small scale eddies in the hydrogen jet and larger scales in the recirculation zones. For the LES approach, the Wall-Adapting Local Eddy-viscosity (WALE) model is applied to capture the sub-grid scale turbulent eddies. This was chosen over the standard Smagorinsky-Lilly model due to its improved ability to predict the near-wall turbulence effects essential for accurately representing the hydrogen jet [31].

\section{FTF ESTIMATION METHODS}

The linear flame transfer function represents the response of the unsteady heat release rate to small perturbations in the inlet flow velocity [32]. This is written in the form:

$$
F T F(\omega)=\frac{Q^{\prime} / \bar{Q}}{u_{i n}^{\prime} / \bar{u}_{i n}}
$$

Where $\omega$ is angular frequency, $Q^{\prime}$ is instantaneous heat release rate fluctuation, $\bar{Q}$ is mean heat release rate, $u_{i n}^{\prime}$ is instantaneous inlet velocity fluctuation and $\bar{u}_{\text {in }}$ is mean inlet velocity.

In this linear case the FTF is purely a function of forcing frequency, with no dependence on the value of the fluctuating velocity. The FTF in this form can be directly measured in experiments by imposing small velocity fluctuations in the flow upstream of the flame and monitoring the heat release in the flame through velocity or pressure sensors combined with chemiluminescence. This can also be achieved using time resolved CFD simulations combined with system identification techniques [33]. Determining the FTF using experiments is often a costly procedure and is not suitable for early development stages. On the other hand computing the FTF directly from CFD can be highly time intensive as the computationally expensive LES approach must be used to accurately predict the flame dynamics. To avoid these issues it is possible to instead use analytical formulations of the FTF based on the driving mechanisms coupling the acoustic field and the unsteady heat release rate fluctuations [34]. These methods do not require a time-varying simulation to estimate the constants for the formulations and can therefore be carried out using relatively fast RANS simulations which makes them ideal for preliminary studies and a potentially as a design tool. This approach does introduce additional modelling compared with the LES method both in terms of the flow prediction and the FTF estimation. As RANS uses a turbulence model for all length scales, it's ability to predict the influence of the jet in cross-flow on the mixing process will likely be negatively impacted. It is also essential that the analytical FTF formulation used captures the main driving mechanism of thermoacoustic instabilities.
TABLE 2: SUMMARY OF FTF ESTIMATION METHODS

\begin{tabular}{ccc}
\hline FTF Name & Simulation & Estimation Method \\
\hline FTF-1 & RANS & Modified $n$-tau model \\
FTF-2 & LES & Single Frequency Velocity Forcing \\
FTF-3 & LES & Multiple Frequency Velocity Forcing \\
\hline
\end{tabular}

Methods based on both RANS and LES CFD simulations are compared in this work which are described in the following and summarised in Table 2.

\section{RANS Based Approach}

The analytical formulations used to estimate the FTF are based on the choice of the most significant driving mechanisms coupling the acoustic field and unsteady heat release fluctuations. This is dependant on several parameters, including the type of fuel, flame structure and operating conditions. The most relevant mechanism for hydrogen micromix combustion is assumed to be equivalence ratio fluctuations which is proportional to perturbations in the inlet velocity.

$$
\frac{Q^{\prime}}{\bar{Q}} \propto \frac{\varphi^{\prime}}{\bar{\varphi}} \propto \frac{u_{i n}^{\prime}}{\bar{u}_{i n}}
$$

Where $\varphi^{\prime}$ is the instantaneous equivalence ratio fluctuation and $\bar{\varphi}$ is the mean equivalence ratio.

This leads to Crocco's $n$-tau model for the FTF shown below [35].

$$
F T F(\omega)=n e^{-i \omega \tau}
$$

Where $n$ is a proportionality constant, which is set to a value of 1 for preliminary studies to adhere to the theoretical low frequency limits of FTFs which indicate that the gain tends to 1 as frequency approaches 0 [36], and $\tau$ is the time delay between the inlet perturbation and the fluctuation in heat release.

This model was originally developed for liquid rocket engines however it is now applied to essentially all combustion systems and is still regarded as a good first approximation of the flame transfer function [37]. The main limitation of this model is the estimation of a constant value of magnitude for all frequencies. As the frequency of the oscillations increases, the flames ability to amplify the disturbances is reduced [38]. This is a result of the increasing dispersion of the perturbation with frequency to such a degree that the flame no longer follows the perturbation and the amplification factor of the flame tends to zero [39]. The 
standard $n$-tau model will instead over predict high frequency oscillations and will therefore provide a conservative estimate for instabilities at high frequencies.

In order to better match with experimental data and represent the effect of reducing gain with frequency, a modification to the $n$-tau model has been developed which accounts for the variation of the time delay due to flow dispersion [40]. In this model the time delay is assumed to vary uniformly from a value of $\tau-\Delta \tau$ to $\tau-\Delta \tau$. This modified formula takes the form:

$$
F T F(\omega)=n \theta e^{-i \omega \tau}
$$

Where the $\Theta$ term represents the influence of the time delay spread on the flame amplification. This term is calculated by:

$$
\theta=\frac{\sin (\omega \Delta \tau)}{\omega \Delta \tau}
$$

Where $\Delta \tau$ is the spread of time delays which provide a measure of the non-uniformity of the flow.

The key parameter which must be determined from the RANS simulations is the flame time delay, $\tau$, which describes the time taken for the flame to respond to acoustic disturbances in the inlet velocity. In this work the time delay is approximated as the mean residence time between the location of fuel injection and the position of the flame front. This represents the time taken for fluctuations in the FAR generated at the fuel injector to propagate into the flame. Using this approximation the time delay can be determined by calculating the mean time taken for particles injected at the fuel injection point to reach a specified location within the flame, while the spread of time delays $\Delta \tau$ can be determined by considering the time taken for each individual injected particle.

To define the location of the flame front, the progress variable, $c$, is used which describes the reaction process as varying from zero where combustion has not occurred to one where the combustion process is complete. The upper range of the progress variable values are increasingly dominated by residual reactions which do not contribute significantly to the heat release, and therefore the flame acoustics. Therefore, although value of $c=1$ represents complete combustion, the most significant portion of the reaction process can be considered to be complete prior to this, in the range of approximately 0.9 to 0.95 . The selection of the value of progress variable which represents the flame front is arbitrary and therefore requires investigation to determine its effect on flame time delay.

In order to measure the mean residence time between the location of fuel injection and position of the flame front, the Discrete Phase Model (DPM) in ANSYS Fluent 18.2 was used. This allows a separate stream of particles to be injected and monitored as they travel trough the domain. This can be applied in postprocessing instead of throughout the simulation to avoid both an increase in computational expense and the influence of the discrete phase particles on the flow field. When applied in this manner the node values of the resolved flow field are used to determine the particle trajectories.

Inert particles were injected from the outlet face of the hydrogen injection pipe. The number of individual injection points is determined by the number of grid nodes present on the defined surface which was found to be 156. A stochastic tracking model was enabled to account for the influence of turbulent velocity fluctuations on the particle trajectories which injects multiple particles per injection point. 10 injections per injection point was selected after an investigation into the sensitivity of the estimated mean time delay to the number of particles injected. This resulted in 1,560 individual particles being injected in total.

\section{LES Based Approach}

The flame transfer function is obtained by directly measuring the response of the unsteady heat release rate to sinusoidal inlet velocity perturbations at various frequencies. The velocity perturbations result in an equivalence ratio fluctuation at the hydrogen injection hole which will propagate into the flame. As the peak of the velocity perturbation will result in a decrease in FAR, there will be a $180^{\circ}$ phase difference between the velocity perturbation and equivalence ratio fluctuation. The sinusoidal form of the perturbation was chosen to imitate the acoustic forcing generated by loudspeakers typically used in forced response experimental cases [41]. This imposed velocity profile is represented by:

$$
u=u_{0}\left[1+A \sin \left(2 \pi f_{n} t\right)\right]
$$

Where $u_{0}$ is the unperturbed inlet velocity, $A$ is the normalised velocity forcing amplitude, $f_{n}$ is the forcing frequency and $t$ is time.

Forcing frequencies were chosen based on two criteria: representing the shape of the gain observed from the modified $n$-tau model while also providing sufficient detail in the lowmid frequency range where the gain is predicted to still be significant.

The amplitude of the oscillations was chosen to be small enough such that the heat release rate perturbations do not experience non-linear effects due to saturation, while still being observable above the local turbulence. A value of $u^{\prime}=0.269 \bar{u}$ is suggested to be sufficiently far from the saturation limit to remain in the linear growth regime [42].

A range of five frequencies was selected based on the FTF obtained by the modified $n$-tau model. The amplitude and frequencies chosen are shown in Table 3. 
TABLE 3: FORCING AMPLITUDE AND FREQUENCIES OF THE INLET VELOCITY PERTURBATIONS

\begin{tabular}{ccccc}
\hline \multicolumn{4}{c}{ Forcing Amplitude (-) } \\
\hline \multicolumn{5}{c}{$0.269 u_{0}$} \\
\hline \multicolumn{4}{c}{ Forcing } & Frequencies (Hz) \\
\hline$f_{1}$ & $f_{2}$ & $f_{3}$ & $f_{4}$ & $f_{5}$ \\
337 & 601 & 1847 & 2129 & 3931 \\
\hline
\end{tabular}

An alternative forcing method has also been investigated in which multiple forcing frequencies are superimposed onto a single velocity perturbation which has been successfully applied in thermoacoustic assessments of premixed flames in industrial gas turbines [43]. This method also influenced the choice of forcing frequencies as it was essential that they were sufficiently distributed in such a way that harmonics of the lower forcing frequencies were not close to any of the higher forcing frequencies. Fig. 2 shows the inlet velocity profiles with increasing timestep for both the $601 \mathrm{~Hz}$ single frequency and the multiple frequency forced cases. The numerical form of the multiple frequency inlet velocity perturbation takes the form:

$$
\begin{array}{r}
u=u_{0}\left[1+A \sin \left(2 \pi f_{1} t+\phi_{1}\right)+A \sin \left(2 \pi f_{2} t+\phi_{2}\right)+\ldots\right. \\
\left.+A \sin \left(2 \pi f_{n} t+\phi_{n}\right)\right]
\end{array}
$$

Where $\phi_{n}$ indicates the phase difference of the $n^{\text {th }}$ forcing frequency. This phase difference was applied to allow distinction between the heat release response to each individual frequency. This approach has been applied for a two frequency harmonic forced case [44] however comparison with the single forcing frequency results will indicate whether this multi-forcing approach produces acceptable results. If so this would allow a considerable reduction in time taken to estimate the full non-linear FDF in future work as only a single simulation containing all forcing frequencies would be required for each perturbation amplitude.

Heat release rate is not a variable available for monitoring by default in Fluent 18.2, therefore two possible approaches have been identified. Firstly, a custom field function for the heat release rate could be developed to monitor the fluctuations. While this approach would also have the added advantage of providing additional insight into the heat release characteristics of hydrogen micromix injectors, it was deemed too time intensive for the scope of this project. The alternative is to apply the approach commonly used in experimental analysis of thermoacoustics which is to instead monitor the fluctuations in $\mathrm{OH}$. This is based on the knowledge that, for a variety of flame types, heat

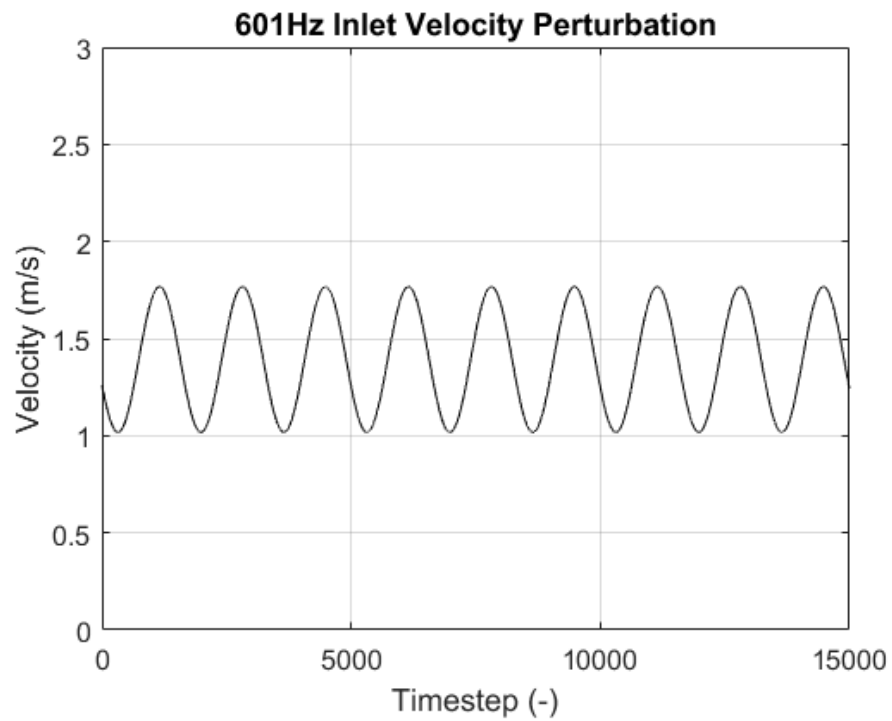

(a)

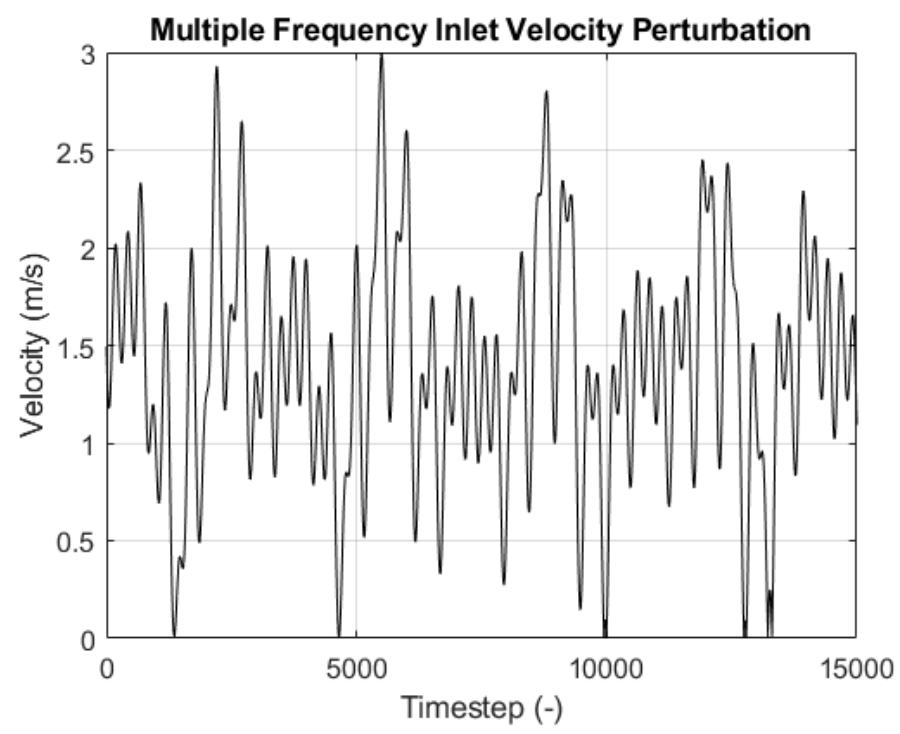

(b)

FIGURE 2: SINUSOIDAL AIR INLET PERTURBATIONS FOR 601HZ SINGLE FORCING CASE (a) AND MULTIPLE FREQUENCY FORCED CASE (b)

release rate fluctuations are approximately proportional to fluctuations in the $\mathrm{OH}$ emissions [45]. Therefore, this assumption can be expressed as:

$$
\frac{Q^{\prime}}{\bar{Q}} \propto \frac{O H^{\prime}}{\overline{O H}}
$$

By estimating a transfer function between the velocity per- 


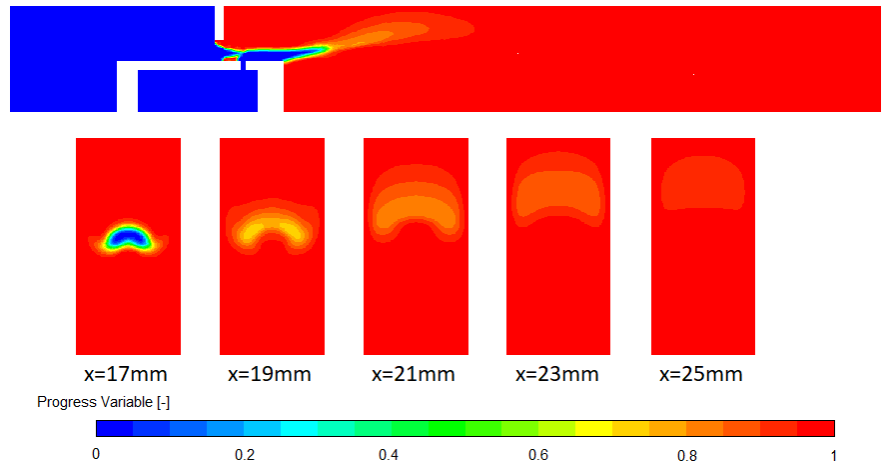

FIGURE 3: CONTOURS OF PROGRESS VARIABLE $c$ ON THE MID-PLANE (UPPER AND AT SPECIFIED AXIAL LOCATIONS (LOWER)

turbation and the $\mathrm{OH}$ fluctuations, the FTF can then be obtained by combining the gain and phase obtained for each frequency and applying a fitting procedure to approximate the continuous form [46]. By determining a series of FTFs in this manner over a range of velocity perturbation amplitudes, the non-linear FDF can be constructed which captures the effects of saturation through the decrease in gain with increasing perturbation amplitude. Due to time constraints and limits on computational resources it was not possible to run the additional simulations required to generate the FDF, however this is an important next step for future research in hydrogen micromix thermoacoustics.

\section{RESULTS AND DISCUSSION RANS Time Delay Estimation}

The contours of progress variable are shown in Fig. 3. This indicates where the reactions are taking place and is used to determine the location of the flame front. The small bubble of reacted flow in the air stream immediately upstream of the hydrogen injection hole does not represent a physical phenomenon but is simply an artefact of the computational model. This is not reflected in the temperature, velocity or species mass fraction contours and has been found to have no significant impact on the rest of the flow domain. It can be seen that the upper and lower reaction regions are compact and the majority of reactions occur within a very short axial distance from injection, which is to be expected of hydrogen micro-diffusion flames. However in the middle of the reaction zone there is a region of residual reactions which extends far downstream. This is likely caused by the low penetration depth of the hydrogen jet which results in the flame being positioned lower in the domain. This extended reaction region occurs at the upper edge of the flame where the FAR is low and close to the flammability limit.

Fig. 4 illustrates the trajectory of the injected particles and the location of the $c=0.9$ contour of the progress variable. Due

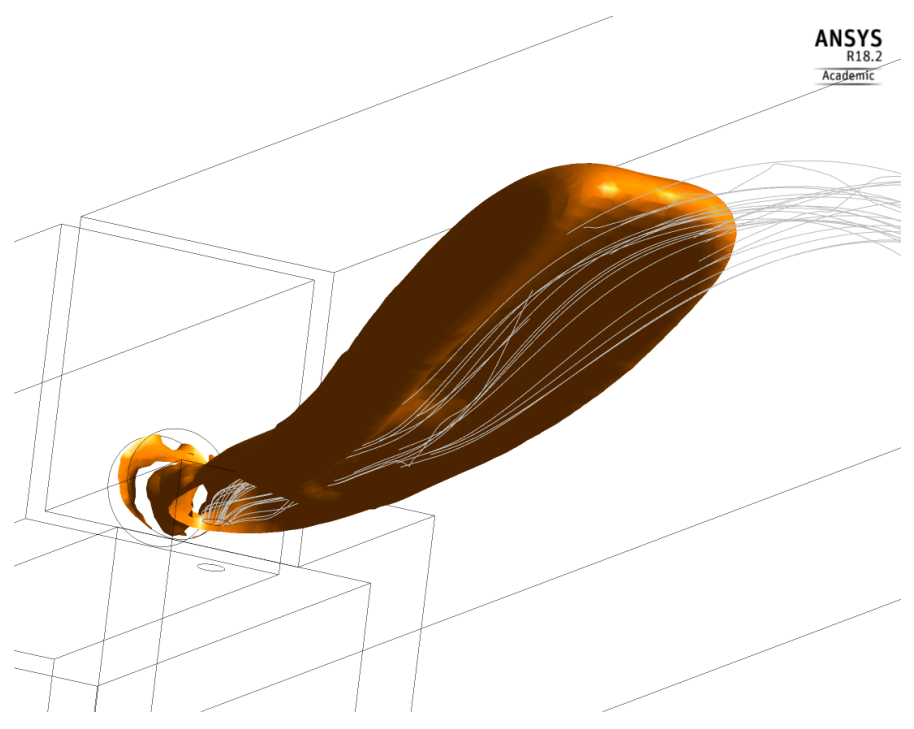

FIGURE 4: DPM PARTICLE TRACKS WITH LOCATION OF THE 0.9 CONTOUR OF PROGRESS VARIABLE

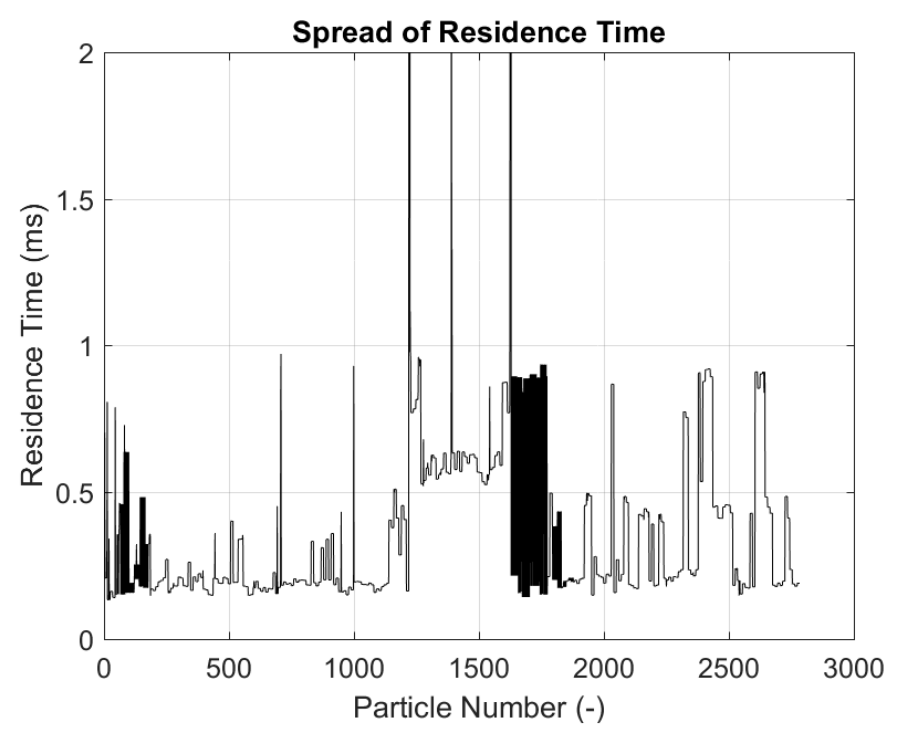

FIGURE 5: COMBINED DATA OR RESIDENCE TIME FOR EACH INJECTED PARTICLE TO REACH BOTH THE $c=0.9$ AND $c=0.95$ CONTOURS OF THE PROGRESS VARIABLE

to the large diffuse region towards the end of the flame and the method by which DPM boundaries must be defined in Fluent 18.2, the residence time was measured between the injection of the DPM particles at the face of the hydrogen injection hole and the surfaces positioned at both the $c=0.9$ and $c=0.95$ contours of the progress variable.

The measured time from injection to both the $c=0.9$ and $c=0.95$ contours is shown in Fig. 5. To estimate the mean time 


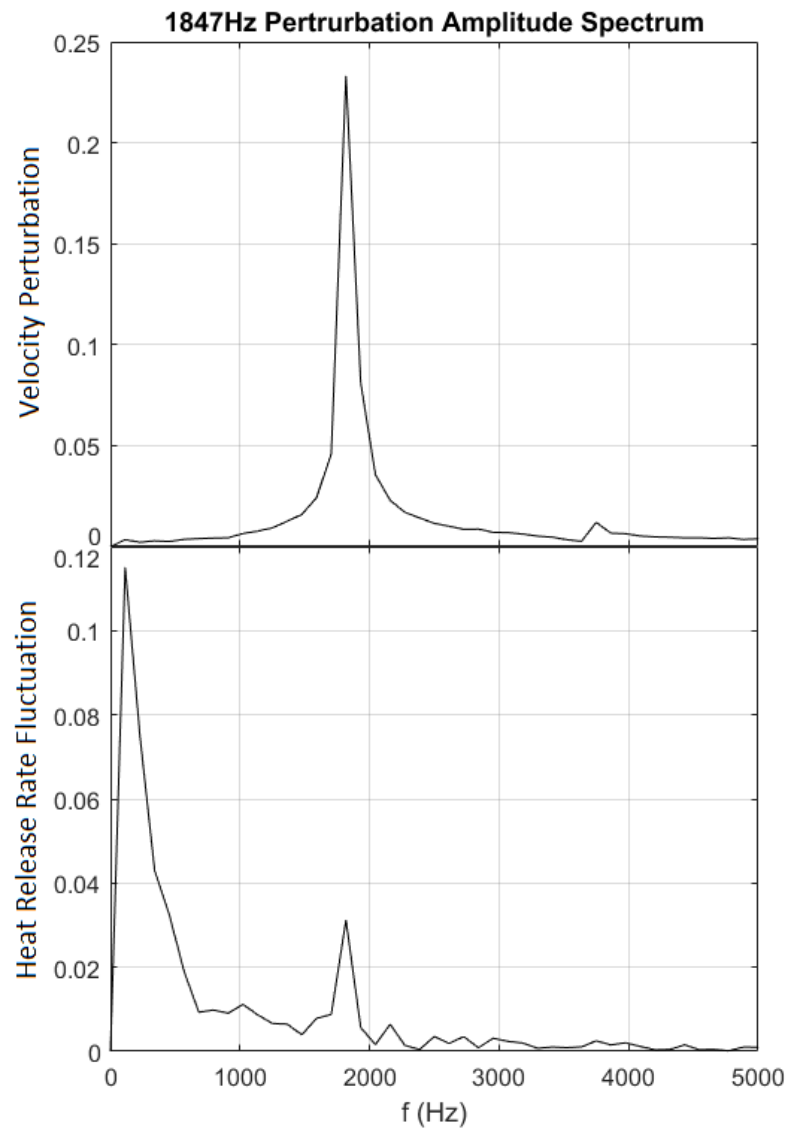

FIGURE 6: FAST FOURIER TRANSFORM (FFT) OF THE NORMALISED FORCED AIR VELOCITY IMMEDIATELY UPSTREAM OF FUEL INJECTION AND THE NORMALISED HEAT RELEASE RATE FLUCTUATIONS FOR THE 1847HZ FORCED CASE

delay, the average of the DPM tracked data is taken. The spread of time delays is represented by \pm one standard deviation of the mean of the tracked particle time delay data to capture the effects of flow dispersion between fuel injection and the flame front.

\section{LES OH Fluctuations}

Multiple simulations were run to cover the range of frequencies described in Table 3. Five simulations were run initially, each with a single forcing frequency. Following this an additional simulation was run with the velocity perturbation containing the five superimposed frequencies.

As described previously the output signal, the $\mathrm{OH}$ mass fraction, was measured over the entire simulation domain. The input signal, the velocity fluctuation, was measured at a point immediately upstream of the hydrogen injection hole within the flow from the air guiding panel to ensure the imposed sinusoidal

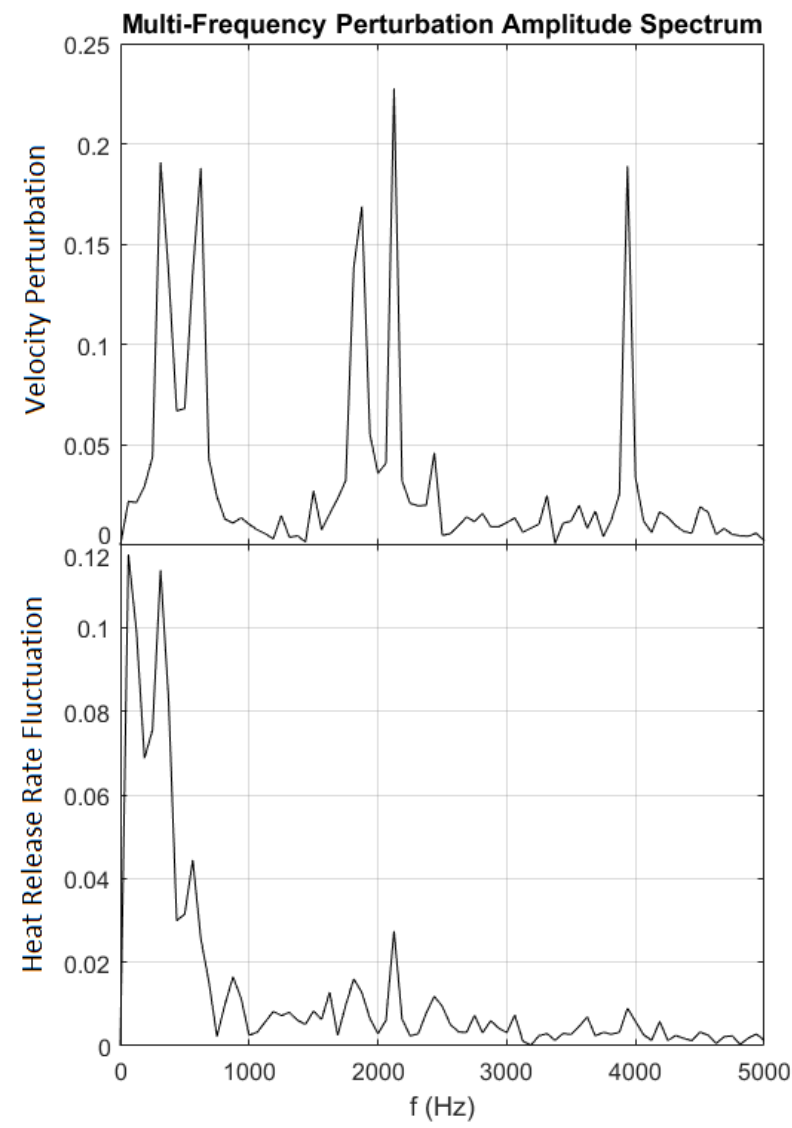

FIGURE 7: FAST FOURIER TRANSFORM (FFT) OF THE NORMALISED FORCED AIR VELOCITY IMMEDIATELY UPSTREAM OF FUEL INJECTION AND THE NORMALISED HEAT RELEASE RATE FLUCTUATIONS FOR THE MULTIPLE FREQUENCY FORCED CASE (b)

perturbation remained visible over the background turbulence. Fig. 6 and Fig. 7 show the Fast Fourier Transform (FFT) of each of these signals for the $1847 \mathrm{~Hz}$ and multiple frequency forced cases respectively. In each case the imposed velocity perturbation is clearly visible over the background turbulence, however the amplitude of the signal has decreased by varying amounts for each frequency.

Peaks are observed in the $\mathrm{OH}$ fluctuation plots at the same frequencies as the velocity forcing. As expected these peaks generally decrease in amplitude with increasing forcing frequency which is seen in the multiple frequency forced case, this trend is also present across the individual single frequency forced cases. A large, low frequency peak is also observed at approximately $60 \mathrm{~Hz}$ which is attributed to the quasi-steady, periodic state of the simulation which is also influenced by the generation of hotspots within the flame. This initial peak is present in all forced cases as well as in the unforced simulation. 

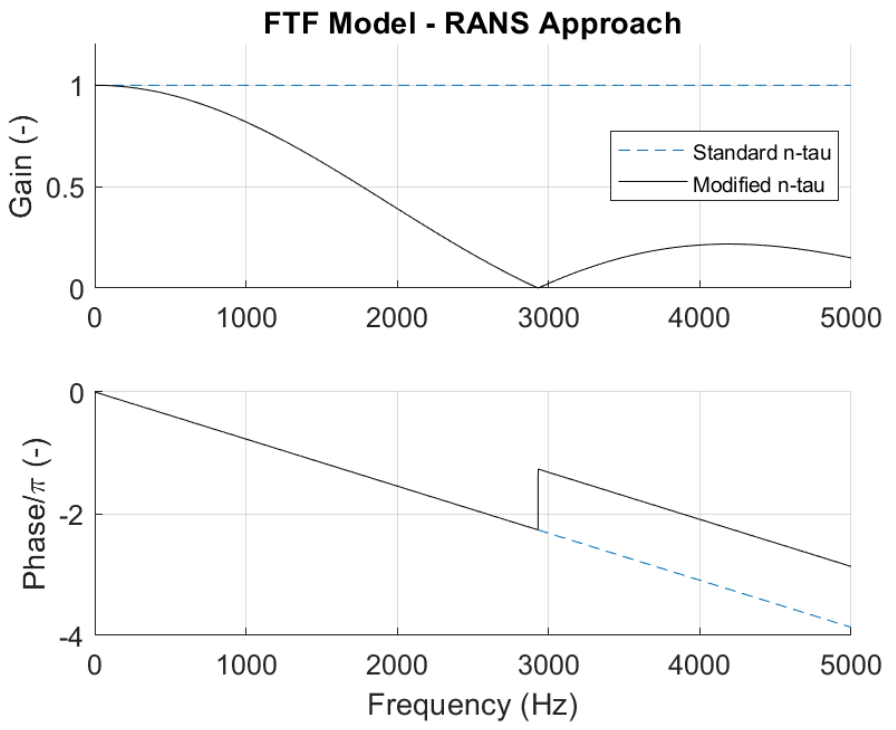

FIGURE 8: FTF-1 OBTAINED FROM RANS CFD COMBINED WITH THE MODIFIED $n$ - tau MODEL WITH STANDARD $n-t a u$ PREDICTION SHOWN FOR COMPARISON

\section{Flame Transfer Functions}

Fig. 8 shows the gain and phase of FTF-1 estimated using the modified $n$-tau model at the time delay and spread of time delays estimated using the RANS simulation. The gain and phase predicted by the standard $n$-tau model for this time delay are also shown for comparison. The modified $n$-tau model is able to predict the low-pass filter behaviour of the flame, with a decrease in gain with frequency to a minimum after which there are a series of declining humps as the gain tends to zero. In practice this minimum between humps does not equal zero [47], however this is acceptable as a first approximation. The $180^{\circ}$ phase shift is also observed at the frequency corresponding to the minimum gain, although in practice the phase shift is less abrupt than in this approximation. Correctly estimating the phase of the FTF is essential as this directly determines whether interactions between the acoustic field and fluctuations in the unsteady heat release rate are constructive, which is a key element of estimating the growth rate of oscillations. The lower theoretical limits for FTFs of these types of flames are observed, which are that gain tends to 1 and phase tends to zero as the frequency approaches 0 . This occurs as a result of the quasi-steady nature in this range such that a perturbation in the inlet flow results in an equal fluctuation in the heat release rate [36].

Compared with FTFs for conventional combustion systems $[49,50]$, an example of which is shown in Fig. 9, the gain for the hydrogen micromix FTF does not decrease significantly until a much higher frequency. The flame is therefore able to continue to amplify oscillations over a much wider frequency range than is possible in diffusion or premixed combustors burning conven-

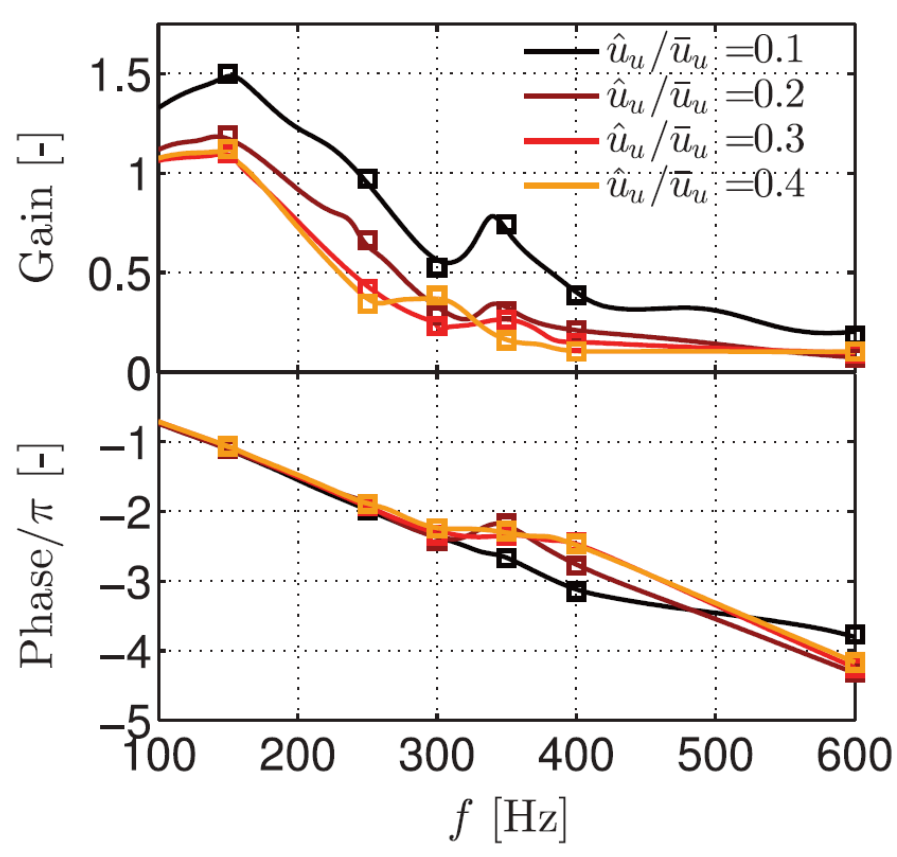

FIGURE 9: FTF FOR A TYPICAL PREMIXED COMBUSTION SYSTEM BURNING AN AIR/ETHYLENE MIXTURE [48]

tional fuels such as kerosene and natural gas. This further reinforces the assertion that thermoacoustic issues cannot be ignored in hydrogen micromix systems.

Shown in Fig. 10 is FTF-2 and FTF-3 obtained from the single and multiple frequency forced simulations respectively with a fitting procedure applied using MATLAB. Due to the low number of data points available, corresponding to the 5 simulated frequencies, the data did not necessarily fully capture the form of the FTF. As computational resources were limited, the number of timesteps for which each simulation was run also reduces the accuracy of the transfer functions between each velocity perturbation and the resulting $\mathrm{OH}$ fluctuation.

Both the single and multiple forced cases exhibit the same trends, however as the frequency tends to zero, the gain tends towards 1 and the $337 \mathrm{~Hz}$ forced frequency has a considerably higher gain than in the single frequency forced case. The increase in gain following the minimum between $1,000 \mathrm{~Hz}$ and $2,000 \mathrm{~Hz}$ is also more pronounced. The phase of the fitted FTF does not display the consistently decreasing phase, however this is a limitation of the fitting method.

This is a promising indication that the multiple frequency forcing method is capable of estimating the FTF to a similar degree of accuracy as a series of single frequency forced simulations. However, due to the increased background noise seen in Fig. 7 it is recommended to increase the perturbation amplitude so the fluctuations at high forcing frequencies are more clearly 


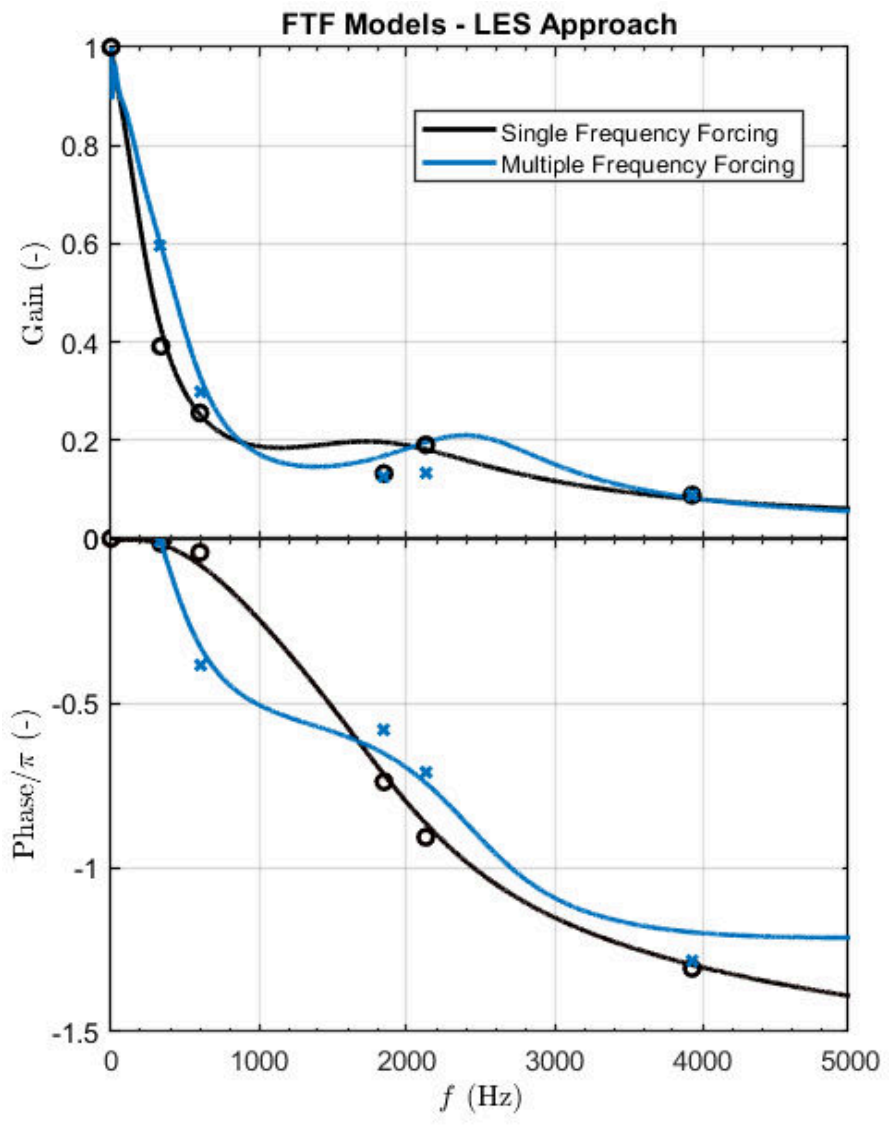

FIGURE 10: FTF-2 (SINGLE FREQUENCY FORCING) AND FTF-3 (MULTI FREQUENCY FORCING) OBTAINED FROM THE SINGLE AND MULTIPLE FREQUENCY FORCED LES SIMULATIONS RESPECTIVELY

visible. The amplitude limit beyond which non-linear effects due to saturation begin to influence the flame acoustic behaviour must be considered when modifying the perturbation amplitude. By using this multiple frequency forcing method, the number of simulations required to estimate the FTF with sufficient resolution over the frequency range of interest can be greatly reduced. This will be particularly useful for the non-linear FDF estimation which requires several of these FTFs to be obtained and then combined.

It can be observed that the predicted drop in gain for the FTFs estimated from LES occurs much more rapidly than in FTF-1, although the overall shape is preserved with a decrease to a minimum gain followed by a slight increase. The trend indicates that the first gain minimum is located between $1,000 \mathrm{~Hz}$ and $2,000 \mathrm{~Hz}$, therefore additional simulations should also be run in this range to determine the value and frequency at which this occurs. This is at a significantly lower frequency than predicted by FTF-1, indicating that the influence of flow dispersion was likely
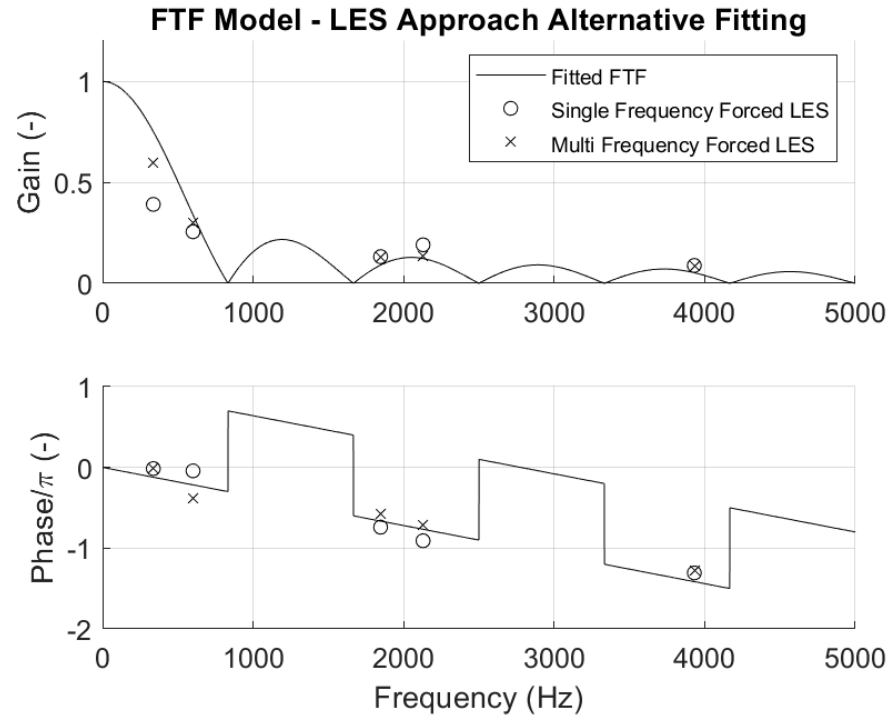

FIGURE 11: ALTERNATIVE FTF FITTING METHOD FOR LES APPROACH USING MODIFIED $n-$ tau MODEL

underestimated and should be further investigated. This could also be an indication that the RANS simulation is unable to accurately capture the jet in cross-flow mixing process or that additional driving mechanisms of heat release fluctuations are present which are not accounted for by the modified $n$-tau model.

The phase also exhibits different behaviour, with a more shallow decrease as frequency increases, although due to the cyclic nature of phase this may not be representative of the real case where a much steeper phase may be present passing through $0-360^{\circ}$ between the data points. This indicates that the value of time delay calculated from the RANS simulation was slightly overestimated, however due to the high sensitivity of the FTF phase to relatively small changes in time delay this could potentially be resolved by using an improved turbulence modelling approach or a different chemical mechanism for the combustion process.

It is essential that the impact of the limited number of data points on the FTF fitting procedure is not overlooked. The methods which have been applied appear to give a reasonable fit, however alternative forms of the FTF are possible using the same data points. If it is assumed that the modified $n$-tau model represents the correct form of the FTF for example, a credible fit for the LES data points can be obtained by altering the value of the time delay, $\tau$ and spread of time delays, $\Delta \tau$, which produces the form shown in Fig. 11. In this case the time delay is shorter than that predicted from the RANS simulations by approximately a factor of 2 while the spread of time delays is roughly 3 times larger. It is not possible to state whether this provides a more representative fit at this stage, however this illustrates the importance of improving the resolution of the LES FTF with additional fre- 
quency forced simulations in order to capture the flame response over the entire frequency range of interest, particularly with data points on each of the humps shown on the gain to investigate the cyclic nature of the phase.

Overall the FTFs obtained from LES simulations are in broad agreement with the findings of the RANS simulations in terms of the shape of the FTF. The gain remains at higher levels up to higher frequencies than would typically be the case for conventional combustors, however to a lesser degree than indicated by the modified $n$-tau model. Thermoacoustic instabilities are therefore likely to occur at higher frequencies than would be expected from current combustors burning kerosene or natural gas as fuels.

\section{FUTURE DEVELOPMENT OF THE HYDROGEN MI- CROMIX CONCEPT}

As both the numerical simulations and the FTF estimation methods used in this work have not yet been validated experimentally for hydrogen micromix combustion, this is the next key step in the development of this concept. Experiments are planned at Cranfield University for both single and multiple injector configurations to validate the numerical approaches. Simulations will also be applied to multiple injector configurations to investigate the influence of injector-injector interactions. For the initial numerical investigations into the multiple injector configuration a 3x3 array is proposed where the central element will be representative of an injector in a full combustor. Validation of the FTF estimation methods used in this work will be achieved by comparing computationally and experimentally obtained FTFs. Following this the thermoacoustic analysis should move towards determining the full non-linear FDF for both single and multiple injector configurations. These FTFs and FDFs will be used in conjunction with low order acoustic models of the system to determine the thermoacoustic stability of both test and engine realistic conditions.

Continued investigation into the thermoacoustics of hydrogen micromix combustion systems in addition to the overall development of the concept will be conducted as part of the ENABLing cryogEnic Hydrogen based CO2 free air transport (ENABLEH2) [51] project coordinated by Cranfield University.

\section{CONCLUSIONS}

Research on the development of hydrogen micromix combustion systems is currently ongoing at Cranfield University both through numerical simulation and experiments with the goal of simulation and testing of a full annular combustor. The present work provides an initial insight into the thermoacoustic properties of hydrogen micromix flames and a foundation for future publications regarding numerical and experimental determination of flame response and the application of low-order acoustic modelling to both rig and engine-realistic combustor configurations.

FTFs for a single hydrogen micromix injector element have been estimated using computational approaches, based on both RANS CFD simulations combined with analytical formulations and LES CFD. At present these methods have not yet been validated experimentally but are intended to inform the design of the micromix concept and upcoming experimental tests of the likely thermoacoustic characteristics of these combustion systems.

For the RANS based approach, the FTF was estimated using a modification to the $n$-tau model which accounts for the non-uniformity of the flow. The key finding of this approach is that the modified $n-t a u$ model predicts the FTF gain will remain at high values up to significantly higher frequencies than is typically observed in conventional combustion systems. This suggests that hydrogen micromix combustors may be prone to higher frequency thermoacoustic instabilities which will be an important consideration for future development of the concept. The risk of thermoacoustic instabilities should therefore not be neglected for hydrogen micromix combustion systems and further studies in this area are recommended to avoid setbacks in later development stages.

FTFs estimated from the LES based approach have been obtained from a series of simulations each containing a different frequency velocity perturbation as well as from a single simulation with the various frequencies superimposed onto a single perturbation. The methods differ in the low frequency behaviour of the FTF however this is believed to be due to both the poor resolution of the transfer functions obtained and the limited number of velocity perturbation frequencies which could be simulated as well as the applied fitting method. Aside from this discrepancy there is good agreement between the two approaches which is a promising indication that the multiple forcing frequency method is able to provide a representative estimate of the FTF which will greatly reduce the computational expense of thermoacoustic analyses using LES.

The FTF estimated from the RANS approach is in broad agreement with those estimated from the LES approach in terms of the shape of the FTF, however the drop in gain occurs far more rapidly in the LES methods. This indicates that with modifications to the method of obtaining the constants from the RANS simulations for use in the analytical formulations, this lower fidelity approach to FTF estimation could potentially be used as a preliminary design tool for the development of the hydrogen micromix concept due to its significantly lower computational expense compared with LES simulations.

\section{ACKNOWLEDGEMENTS}

This ENABLEH2 project is receiving funding from the European Union's Horizon 2020 research and innovation programme under grant agreement No 769241. 


\section{REFERENCES}

[1] Lieuwen, T. C., and Yang, V., 2013. Gas Turbine Emissions. Cambridge University Press, Cambridge, UK.

[2] Lefebvre, A. H., and Ballal, D. R., 2010. Gas Turbine Combustion: Alternative Fuels and Emissions, 3rd ed. CRC Press, Boca Raton, Florida.

[3] Liu, Y., Sun, X., Sethi, V., Nalianda, D., Li, Y. G., and Wang, L., 2017. "Review of Modern Low Emissions Combustion Technologies for Aero Gas Turbine Engines". Progress in Aerospace Sciences, 94, pp. 12-45.

[4] Johnson, T., Cooper, J., Petsonk, A., Zeinali, M., and Hemmings, B., 2012. A New Flightplan: Getting Climate Measures for Aviation off the Ground.

[5] Shafiee, S., and Topal, E., 2009. "When will Fossil Fuel Reserves be Diminished?”. Energy Policy, 37(1), pp. 181189.

[6] Khandelwal, B., Karakurt, A., Sekaran, P. R., Sethi, V., and Singh, R., 2013. "Hydrogen Powered Aircraft: The Future of Air Transport". Progress in Aerospace Sciences, 60, pp. 45-59.

[7] Goodger, E., 2017. Hydrogen as a Transport Fuel. Landfall Press.

[8] Miyamoto, K., Inoue, K., Kawakami, T., Nakamura, S., Tanimura, S., and Masada, J., 2018. "Development of Hydrogen and Natural Gas Co-firing Gas Turbine". In The Future of Gas Turbine Technology 9th International Gas Turbine Conference.

[9] Cecere, D., Giacomazzi, E., and Ingenito, A., 2014. "A Review on Hydrogen Industrial Aerospace Applications". International Journal of Hydrogen Energy, 39(20), pp. 10731-10747.

[10] Lee, H., Hernandez, S., McDonell, V., Steinthorsson, E., Mansour, A., and Hollon, B., 2009. "Development of Flashback Resistant Low-Emission Micro-Mixing Fuel Injector for $100 \%$ Hydrogen and Syngas Fuels". In ASME Turbo Expo 2009: Power for Land, Sea and Air, pp. 411419.

[11] Karagozian, A. R., 2014. "The Jet in Crossflow". Physics of Fluids, 26.

[12] Correa, S. M., 1998. "Power Generation and Aeropropulsion Gas Turbines: From Combustion Science to Combustion Technology". In 27th Symposium (International) on Combustion, pp. 1793-1807.

[13] Esmaeelzade, G., Khani, M. R., Riazi, R., and Sabour, M. H., 2017. "Theoretical Analysis of the Conical Premixed Flame Response to Upstream Velocity Disturbances Considering Flame Speed Development Effects". Propulsion and Power Research, 6(1), pp. 70-78.

[14] Abbott, D. J., Bowers, J. P., and James, S. R., 2012. "The Impact of Natural Gas Composition Variations on the Operation of Gas Turbines for Power Generation". In 6th International Conference for The Future of Gas Turbine Tech- nology.

[15] Yang, D., and Morgans, A. S., 2016. "A Semi-Analytical Model for the Acoustic Impedance of Finite Length Circular Holes with Mean Flow". Journal of Sound and Vibration, 384, pp. 294-311.

[16] Han, X., and Morgans, A. S., 2015. "Simulation of the Flame Describing Function of a Turbulent Premixed Flame using an Open-Source LES Solver". Combustion and Flame, 162(5), pp. 1778-1792.

[17] Murthy, P., 2011. "Numerical Study of Hydrogen Micromix Combustors for Aero Gas Turbine Engines". MSc Thesis, Cranfield University.

[18] Karakurt, A., 2013. "Parametric Investigation of Combustion Characteristics of a Hydrogen Micromix Combustor Concept". MSc Thesis, Cranfield University.

[19] Petit de Bantel, M., 2014. "Comparison Study of Combustion Models for a Conceptual Hydrogen Micromix Combustor". MSc Thesis, Cranfield University.

[20] Degroote, A. J. G., 2014. "Assessment of the Feasability of Controlling the Outlet Temperature Distribution of a Novel Micromix Combustor". MSc Thesis, Cranfield University.

[21] Asanitthong, S., 2014. "Outlet Temperature Distribution Control and Heat Transfer Calculation for a Hydrogen Micromix Combustor". MSc Thesis, Cranfield University.

[22] Sabin, R., 2016. "Comparative Performance Study of CFD Species Models for Hydrogen Micromix Combustion”. MSc Thesis, Cranfield University.

[23] Leon, H. J. A., 2016. "Assessment of the Feasability of Controlling Hydrogen Injection Penetration in a Novel Micromix Combustor". MSc Thesis, Cranfield University.

[24] Abdallah, R. B., 2017. "Ultra-low NOx Micromix Hydrogen Combustion System: -Preliminary Design and Performance Assessment of Novel Injectors Using RANS and LES CFD". MSc Thesis, Cranfield University.

[25] Babazzi, G., 2018. "Turbulence-Chemistry Interaction Study for a Hydrogen Micromix Combustion System". MSc Thesis, Cranfield University.

[26] McClure, J., 2018. "Preliminary Thermoacoustic Risk Assessment for Hydrogen Micromix Combustion Systems using CFD". MSc Thesis, Cranfield University.

[27] Abdallah, R. B., Sethi, V., Gauthier, P. Q., Rolt, A., and Abbott, D., 2018. "A Detailed Analytical Study of Hydrogen Reaction in a Novel Micromix Combustion System". In ASME Turbo Expo 2018: Turbomachinery Technical Conference and Exposition.

[28] Funke, H. H. W., Keinz, J., Kusterer, K., Haj Ayed, A., Kazari, M., Kitajima, J., Horikawa, A., and Okada, K., 2017. "Experimental and Numerical Study on Optimizing the DLN Micromix Hydrogen Combustion Principle for Industrial Gas Turbine Applications". Journal of Thermal Science and Engineering Applications, 9, p. 2017.

[29] Haj Ayed, A., Kusterer, K., Funke, H. H. W., Keinz, J., Str- 
iegan, C., and Bohn, D., 2015. "Improvement Study for the Dry-Low-NOx Hydrogen Micromix Combustion Technology". Propulsion and Power Research, 4(3), pp. 132-140.

[30] Babazzi, G., Gauthier, P. Q., Agarwal, P., McClure, J., and Sethi, V., 2019. "Turbulence-Chemistry Interaction Study for a Hydrogen Micromix Combustion System". In ASME Turbo Expo 2019: Turbomachinery Technical Conference and Exposition.

[31] ANSYS Inc, 2013. Fluent 15.0 Theory Guide.

[32] Cuquel, A., Durox, D., and Schuller, T., 2011. "Theoretical and Experimental Determination of the Transfer Function of a Laminar Premixed Flame". In Proceedings of the Combustion Institute.

[33] Chong, L. T., Komarek, T., Kaess, R., Stephan, F., and Polifke, W., 2010. "Identification of Flame Transfer Functions from LES of a Premixed Swirl Burner". In ASME Turbo Expo 2010: Power for Land, Sea and Air.

[34] You, D., Sun, X., and Yang, V., 2003. "A ThreeDemensional Linear Acoustic Analysis of Gas Turbine Combustion Instability”. In 41st Aerospace Sciences Meeting and Exhibit.

[35] Crocco, L., and Cheng, S.-I., 1956. Theory of Combustion Instability in Liquid Propellant Rocket Motors, Vol. 21. Butterworths Scientific Publications, New York City, New York.

[36] Polifke, W., and Lawn, C., 2007. "On the Low-Frequency Limit of Flame Transfer Functions". Combustion and Flame, 151, pp. 437-451.

[37] Lieuwen, T. C., and Yang, V., 2006. Combustion Instabilities In Gas Turbine Engines: Operational Experience, Fundamental Mechanisms, and Modeling. AIAA, Arlington, Texas.

[38] Dowling, A. P., and Mahmoudi, Y., 2015. "Combustion Noise". Proceedings of the Combustion Institute, 35(1), pp. 65-100.

[39] Innocenti, A., Andreini, A., and Facchini, B., 2015. "Numerical Identification of a Premixed Flame Transfer Function and Stability Analysis of a Lean Burn Combustor". Energy Procedia, 82, pp. 358-365.

[40] Sattelmayer, T., 2003. "Influence of the Combustor Aerodynamics on Combustion Instabilities From Equivalence Ratio Fluctuations". Journal of Engineering for Gas Turbines and Power, 125(1), pp. 11-19.

[41] Paschereit, C. O., Schuermans, B., Polifke, W., and Mattson, O., 2002. "Measurement of Transfer Matrices and Source Terms of Premixed Flames". Journal of Engineering for Gas Turbines and Power, 124(2), p. 239.

[42] Palies, P., Durox, D., Schuller, T., and Candel, S., 2011. "Nonlinear Combustion Instability Analysis based on the Flame Describing Function Applied to Turbulent Premixed Swirling Flames". Combustion and Flame, 158, pp. 19801991.
[43] Rofi, L., Campa, G., Anisimov, V., Dacc, F., Bertolotto, E., Gottardo, E., and Bonzani, F., 2018. "Numerical Procedure for the Investigation fo Combustion Dynamics in Industrial Gas Turbines: LES, RANS and Thermoacoustics". In ASME Turbo Expo 2015: Turbine Technical Conference and Exposition.

[44] Han, X., Yang, J., and Mao, J., 2016. "LES Investigation of Two Frequency Effects on Acoustically Forced Premixed Flame". Fuel, 185, pp. 449-459.

[45] Nori, V., and Seitzman, J., 2008. "Evaluation of Chemiluminescence as a Combustion Diagnostic under Varying Operating Conditions". In 46th AIAA Aerospace Sciences Meeting and Exhibit.

[46] Li, J., Yang, D., Luzzato, C., and Morgans, A. S., 2017. Open Source Combustion Instability Low Order Simulator (OSCILOS-Long) Technical Report.

[47] Tay-Wo-Chong, L., Bomberg, S., Ulhaq, A., Komarek, T., and Polifke, W., 2012. "Comparative Validation Study on Identification of Premixed Flame Transfer Function". Journal of Engineering for Gas Turbines and Power, 134.

[48] Han, X., Li, J., and Morgans, A. S., 2015. "Prediction of Combustion Instability Limit Cycle Oscillations by Combining Flame Describing Function Simulations with a Thermoacoustic Network Model". Combustion and Flame, 162(10), pp. 3632-3647.

[49] Andreini, A., Facchini, B., Giusti, A., and Turrini, F., 2014. "Assessment of Flame Transfer Function Formulations for the Thermoacoustic Analysis of Lean Burn Aero-Engine Combustors". Energy Procedia, 45, pp. 1422-1431.

[50] Armitage, C. a., Riley, A. J., Cant, R. S., Dowling, A. P., and Stow, S. R., 2004. "Flame Transfer Function for Swirled LPP Combustion From Experiments and CFD". In ASME Turbo Expo 2004: Power for Land, Sea and Air.

[51] EU Horizon 2020 Project, 2018. ENABLing cryogEnic Hydrogen based $\mathrm{CO} 2$ free air transport (ENABLEH2) Project, available at https://cordis.europa.eu/project/rcn/216008_en.html. 
2019-11-05

\section{Comparison of hydrogen micromix flame transfer functions determined using RANS and LES}

McClure, Jonathan

ASME

McClure J, Abbott D, Agarwal P, et al., (2019) Comparison of hydrogen micromix flame transfer functions determined using RANS and LES. In: ASME Turbo Expo 2019, 17-21 June 2019,

Phoenix, Arizona, USA, Paper number GT2019-90538

https;//doi.org/10.1115/GT2019-90538

Downloaded from Cranfield Library Services E-Repository 